\title{
Resveratrolo e diabete: dagli studi animali a quelli umani
}

\author{
Marco Centanni • Nunzia Brusca
}

Pubblicato online: 20 gennaio 2015

(C) Springer International Publishing AG 2015

Commento a:

Resveratrol and diabetes: from animal to human studies.

T. Szkudelski, K. Szkudelska.

Biochim Biophys Acta (2014)

doi:10.1016/j.bbadis.2014.10.013

Il resveratrolo è un polifenolo naturale con proprietà antiossidanti, antinfiammatorie, cardio- e neuroprotettive e antitumorali. Esso è presente in alimenti comuni quali i frutti rossi, le arachidi, l'uva e, conseguentemente, il vino rosso. Il ruolo di questo antiossidante è stato particolarmente studiato nel diabete mellito e nelle relative complicanze metaboliche, in modelli umani e animali. Questa review di Szkudelski focalizza i molteplici aspetti dell'azione del resveratrolo sui meccanismi che controllano l'omeostasi glucidica. Il polifenolo svolge un ruolo protettivo sulle isole pancreatiche aumentando la sintesi di enzimi antiossidanti, quali la superossido-dismutasi, la glutatione perossidasi e la glutatione-s-transferasi, che contrastano l'azione dei radicali liberi. Il potere antiapoptotico nei confronti delle $\beta$-cellule è emerso sia nei modelli animali con danno tossico da streptozotocina, sia nell'insulite autoimmune del diabete mellito tipo 1 , ove l'azione del resveratrolo si manifesta riducendo l'espressione del recettore della chemochina 6 e inibendo la migrazione delle cellule infiammatorie nel pancreas. Il resveratrolo, inoltre, modula l'omeostasi glicemica a livello epatico, riducendo l'attività degli enzimi della gluconeogenesi e aumentando, al contrario, quella della glicogeno-

M. Centanni $(\varangle) \cdot$ N. Brusca

Dipartimento di Scienze e Biotecnologie Medico-Chirurgiche, UOC di Endocrinologia, "Sapienza" Università di Roma, Latina, Italia

e-mail: marco.centanni@uniroma1.it sintetasi. A livello muscolare, oltre che epatico, l'ottimizzazione del metabolismo degli acidi grassi e la riduzione dell'espressione di NF-kB e delle citochine proinfiammatorie negli organi bersaglio, sono riconducibili a un' azione diretta del polifenolo, che induce una parziale rigenerazione delle $\beta$-cellule e determina un aumento della concentrazione plasmatica di insulina. Nel diabete mellito di tipo 2, il resveratrolo contrasta la resistenza insulinica nei ratti alimentati con diete iperlipidiche e ad alto contenuto di fruttosio come pure in quelli con insulino-resistenza geneticamente determinata. Nei ratti obesi, infatti, il polifenolo contrasta l'adipogenesi e riduce l'infiltrato macrofagico nel tessuto adiposo, principale sorgente delle adiponectine, corresponsabili della comparsa di insulino-resistenza. Inoltre, il polifenolo determina la riduzione del contenuto lipidico muscolare ed epatico. Questi effetti conseguono alla modulazione dell'azione di due importanti regolatori intracellulari, un'istone deacetilasi (SIRT1) e una chinasi AMP-dipendente (AMPK), che controllano funzioni cellulari determinanti quali il metabolismo energetico intracellulare, la funzione mitocondriale e l'apoptosi. Questi dati sono stati confermati anche nei primati e negli uomini obesi ove, il trattamento cronico con resveratrolo sembra migliorare la sensibilità insulinica.

In conclusione, l'azione pleiotropica del resveratrolo fa di questo polifenolo una possibile risorsa naturale aggiuntiva nel trattamento del paziente diabetico. Il suo effetto benefico si manifesta attraverso l'incremento della secrezione insulinica, la riduzione dell'insulino-resistenza e la soppressione della gluconeogenesi epatica. Inoltre, l'efficacia del resveratrolo nel mitigare la distruzione autoimmune delle $\beta$-cellule pancreatiche è particolarmente rilevante dal punto di vista clinico perché potrebbe rappresentare un supporto al trattamento convenzionale del paziente con diabete mellito tipo 1. 